\title{
Study of Electromagnetic Disruption Forces for Plasma Detachment Measurements in DEMO
}

\author{
Samir El Shawish \\ Jožef Stefan Institute, Jamova 39, SI-1000, Ljubljana, Slovenia \\ Louis Giannone, Arne Kallenbach \\ Max-Planck-Institut für Plasmaphysik, Boltzmannstrasse 2, D-85748 Garching, Germany
}

\begin{abstract}
Global plasma instabilities such as plasma disruptions and vertical displacement events (VDEs) can generate huge transient electrical currents in the conductive parts of the divertor. The interaction of the currents with the magnetic field induces Lorentz force loads. Particularly, the force induced by a VDE can impose a critical impact on the divertor water-cooling pipes due to high current density. A recently proposed isolated-target design solution for the DEMO divertor introduces a shunt element between the target plate and the cassette body to divert electrical current towards more massive cassette body. Such a design solution is also considered as a diagnostic tool for measuring thermo currents (voltages) through a shunt resistor to control plasma detachment.

In this study, a finite-element analysis is performed to provide a conservative upper value for the shunt resistance by investigating the impact of the electromagnetic disruption forces on the integrity of water-cooling pipes in the isolated-target design solution of the DEMO divertor. In the analysis, a maximum allowed pipe current is estimated assuming realistic material properties and magnetic fields, and true geometries of the divertor cassette and water-cooling pipes fixed to the cassette. The results show that local pipe yielding (representing damage initiation) develops for electrical currents larger than $\sim 3.5 \mathrm{kA}$, which sets a conservative (assuming extreme VDE halo currents) upper value for the shunt resistance to $\sim 40 \mu \Omega$.
\end{abstract}

Keywords:

thermo current, shunt resistance, electromagnetic forces, divertor, DEMO 


\section{INTRODUCTION}

In DEMO, the target plates of the divertor must be protected by limiting the heat load to values permitting safe operation. In ASDEX Upgrade, this is achieved by measuring the thermo-current flowing to the target plates as the voltage drop across a shunt resistor and using impurity gas puffing to reduce the heat load to the targets $[1,2]$.

On JET, in a diverted plasma with grounded target plates, current flow between the target plates was measured for the first time [3]. This current is driven by a temperature difference between the inboard and outboard target plates which cause a current flow to compensate the difference in the sheath potentials. A schematic diagram of the potentials along a field line between the target plates has been published [4]. The temperature in front of the inner divertor target is $T_{A}$ and the temperature in front of the outer divertor target is $T_{B}$ with $T_{A}<T_{B}$. It was noted that the thermoelectric current is carried by electrons flowing force-free along the field lines from the colder inner divertor towards the hotter outer divertor. A detailed model of the scrape-off layer outside the separatrix of a diverted tokamak plasma to calculate the current flowing into the divertor plates has been developed [5]. In this model, the parallel thermo-current can also be expressed in terms of the ratio of the power fluxes to the target plates and the ratio of the temperatures at the pre-sheath in front of the target plate. This model also has been extended to include an electron pressure imbalance between the strike zones [6].

For an estimate of the thermo-current in DEMO the following simplified model was applied. The total scrape-off layer resistance is calculated using the resistivity, $\eta_{\|}$,

$$
\eta_{\|}=6.8 \times 10^{-4} T_{e}^{-3 / 2} Z_{\text {eff }}^{0.78}(\Omega m)
$$

where the electron temperature, $T_{e}$, is measured in $\mathrm{eV}$. The $Z_{\text {eff }}$ dependence is an approximation valid within the range occurring in the scrape-off layer

$\left(1<Z_{\text {eff }}<4\right)$. For constant pressure along the field line and assuming equal ion and electron temperatures, the expected parallel net current density, $J_{\|}$, between the target plates is given by (Eq. (17) of [5])

$$
J_{\|}=-\frac{T_{A}}{\bar{\eta}_{\|} L_{\|}}\left(\left(\kappa^{\prime} \frac{T_{B}}{T_{A}}-1\right)+\ln \left\{\frac{1+J_{\|} / J_{\text {sat } A}}{\left[1-\sqrt{T_{B} / T_{A}} J_{\|} / J_{\text {sat } A}\right]^{T_{B} / T_{A}}}\right\}\right)\left(A / m^{2}\right)
$$


where $T_{A}$ has the dimension of Volt, $J_{\text {sat } A}$ is the ion saturation current density at the inner divertor, $\mathrm{L}_{\|}$is the connection length between the targets and the coefficient $\kappa^{\prime}$ contains the different contributions to the net thermoelectric voltage [4].

An upper bound of the expected thermo-current, at the onset of detachment, is calculated with an onion-skin like 2-point model using an up-scaled ASDEX Upgrade SOL geometry. The electron temperature profiles are assumed flat along the target with electron temperatures of $1.5 \mathrm{eV}$ and $5 \mathrm{eV}$ at the inner and outer target, respectively. The electron temperature parallel to the field lines is calculated using Spitzer conductivity and given power flux and mid-plane power and density decay lengths. The ion saturation current at the inner target is estimated from the assumed temperature at the inner divertor and the electron density taken from pressure balance, assuming Mach=1 and an ad hoc momentum loss factor. The average parallel conductivity is calculated using the electron temperature along the field line and assuming $Z_{\text {eff }}=2$ everywhere.

$J_{\|}$is finally obtained from Eq. (2) by numerically searching for the $J_{\|}$ value for which both sides of the equation are equal, taking advantage of the physics argument that $J_{\|} / J_{\text {satA }}$ has to be in the interval 0 to -1 .

Radiation and other divertor dissipation processes are not taken into account in this very simple model, therefore a reduced power across the separatrix is used. Using this model and assuming a power flow of $50 \mathrm{MW}$ at the separatrix, a major tokamak radius of $8 \mathrm{~m}$ and a scrape-off layer power fall-off length of $2 \mathrm{~mm}$, the thermo-current in DEMO is estimated to have a steady state value of approximately $7 \mathrm{kA}$ when plasma detachment begins or, approximately, 140 A per divertor module assuming a divertor design with 48 modules [7]. With strong detachment expected to be mandatory in DEMO, the obtained thermo-current estimate can be regarded as an upper bound estimate.

In the case of vertical displacement events (VDEs), however, the induced halo currents through the divertor can be significantly larger. A conservative estimation follows from the nominal plasma current of 19.6 MA redistributed evenly over 48 divertor cassette modulus. In this extreme limit, the maximum VDE current of $410 \mathrm{kA}$ per divertor can be assumed.

The currently considered design option for thermo-current measurement in DEMO is an isolated-target solution [8] which involves mounting of an isolated target plate so that the applied current flows partially through a tube of resistive material (a shunt) towards the cassette body and partially 


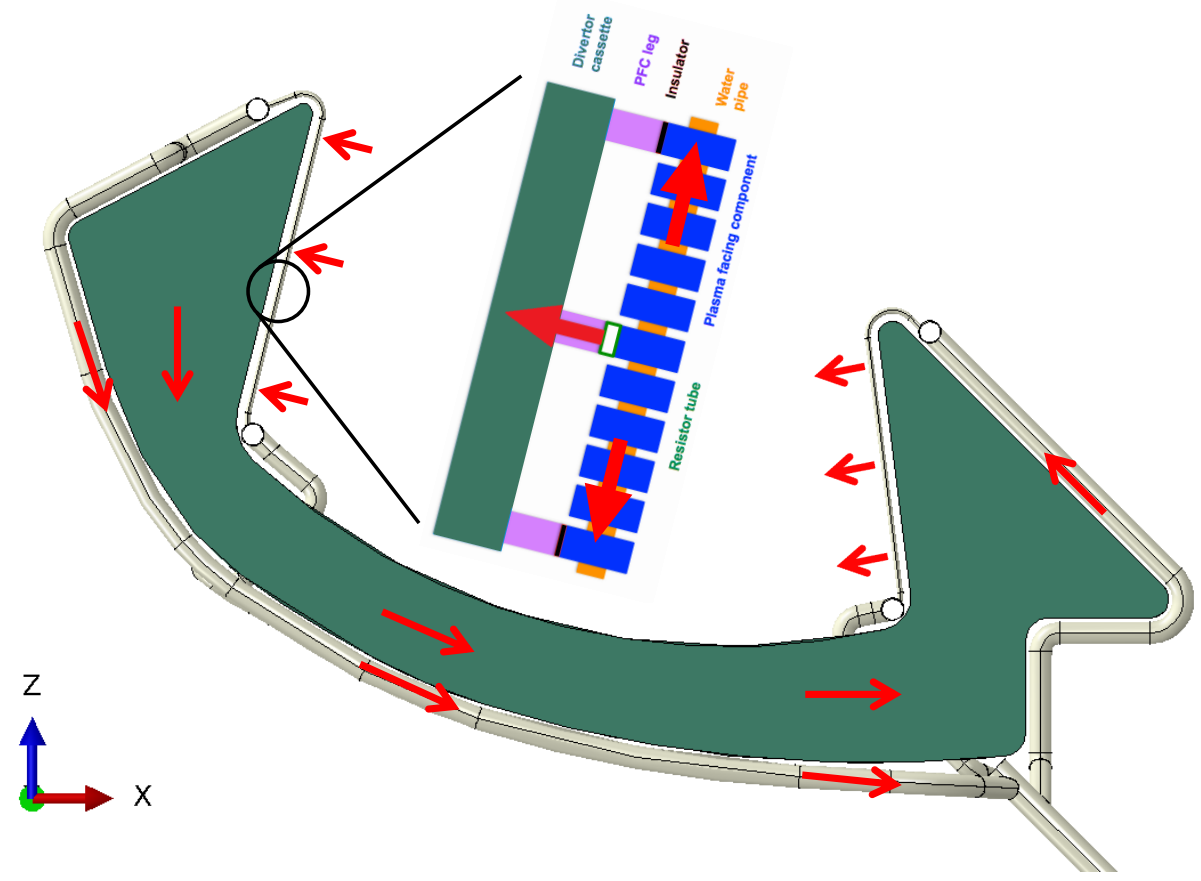

Figure 1: Schematic diagram of the current flow (in red) during thermo-current measurement or during disruption event. It is assumed that plasma facing components (PFC) (in blue) and water-cooling pipes are electrically connected with the divertor cassette only via single resistive element - a shunt (in green). The enlarged part of the scheme is reproduced from Ref. [8].

through the water-cooling pipes as shown in Fig. 1. To maximize the signal to noise ratio for the measured shunt voltage (see Fig. 2),

$$
U_{s}=I R_{p}\left(1+\frac{R_{c}+R_{p}}{R_{s}}\right)^{-1},
$$

the shunt resistance $R_{s}$ should be as large as possible (e.g., $R_{s} \gg R_{c}+R_{p}$ ), assuming electrical isolation of the water-cooling pipes from the divertor cassette to feed the thermo current through a single resistive element. However, in practice, shunt resistance is chosen to be smaller than the water-cooling pipe resistance between the two target plates [8] estimated to be $R_{p}=7 \mathrm{~m} \Omega$ for a stainless steel cooling pipe (see Sec. 3.1 for more detail). This limitation turns out to be necessary in the event of disruption to protect the 


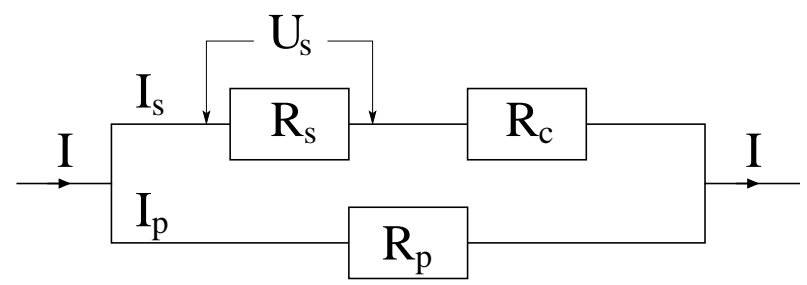

Figure 2: Electrical circuit diagram representing an approximate current flow from Fig. 1: applied current is split into two branches, $I=I_{s}+I_{p}$, and a voltage drop $U_{s}$ is measured over the shunt. $R_{s}, R_{c}$ and $R_{p}$ denote shunt, cassette and water-cooling pipe resistances, respectively. The values of $R_{c}=22 \mu \Omega$ and $R_{p}=7 \mathrm{~m} \Omega$ are calculated in Sec. 3.1.

cooling pipes from high currents and, consequently, high induced mechanical stresses. Namely, the mechanical integrity of the pipes can be jeopardized due to interaction of the currents with external magnetic field (between $\sim 5 \mathrm{~T}$ and $\sim 8 \mathrm{~T}$ at the divertor position in the DEMO tokamak).

The objective of this study is to provide a conservative upper value for the shunt resistance $R_{s, \max }$ in the isolated-target design solution assuming extreme VDE current of $I_{V D E}=410 \mathrm{kA}$ applied to the target plates. The value of $R_{s, \max }$,

$$
R_{s, \max }=R_{p} \frac{I_{p, \max }}{I_{V D E}-I_{p, \max }}-R_{c},
$$

is derived from the requirement that a fraction of the applied current flowing through the water-cooling pipes $I_{p}$ reaches the critical value $I_{p, \max }$ at which (local) damage starts to emerge. In the following, damage initiation is conservatively attributed to the onset of material yielding.

\section{MODEL DEFINITION}

A finite-element (FE) method based 3D electromagnetic analysis is carried out to assess the impact of the induced Lorentz loads and resulting mechanical stresses on the integrity of the water-cooling pipes. The FE modelling and simulations are performed with ABAQUS [9] using isolatedtarget design divertor model. For the assumed electrical connection between the cassette body and the water-cooling pipes through the shunt element, the model is conveniently uncoupled into two sub-models represented by two current paths: (i) path through the cassette body and (ii) path through the water-cooling pipes. The path through the cassette body is used to evaluate 
Table 1: Electrical resistivity and isotropic elasticity data for the materials used in the analysis. The values for electrical resistivity apply for $200^{\circ} \mathrm{C}$, while generic elastic properties are assigned to all the materials.

\begin{tabular}{llll}
\hline Material & Resistivity $(\mu \Omega \mathrm{m})$ & Young's modulus $(\mathrm{GPa})$ & Poisson's ratio $(-)$ \\
\hline Eurofer97 & $0.70[13]$ & 200 & 0.3 \\
AISI-316 & $0.90[14]$ & 200 & 0.3 \\
CuCrZr & $0.04[15]$ & 200 & 0.3 \\
\hline
\end{tabular}

the cassette resistivity $R_{c}$ used in Eq. (4) and to validate the approach with the results published in Ref. [10].

The analysis for each of the current paths is decomposed into two uncoupled simulations. First, a steady-state thermo-electric analysis is performed following Ohm's law (and ignoring thermal effects) to obtain the electrical current distributions $\mathbf{j}(\mathbf{r})$ inside the conductor (the cassette body or the cooling pipes) for the assumed surface current load. In this step, the effect of external magnetic field $\mathbf{B}(\mathbf{r})$ (Hall effect) is neglected. The resulting currents $\mathbf{j}(\mathbf{r})$ are then used to calculate Lorentz magnetic force density field $\mathbf{f}(\mathbf{r})$ at all elements of the FE model using $\mathbf{f}(\mathbf{r})=\mathbf{j}(\mathbf{r}) \times \mathbf{B}(\mathbf{r})$ for $B(r)=B_{0} R_{0} / r$ oriented in toroidal direction. Finally, the $\mathbf{f}(\mathbf{r})$ is employed as a body force load in the second, steady-state linear-elastic mechanical analysis which provides stresses and displacements of the model under assumed boundary conditions.

The proposed strategy follows a similar methodology used in Ref. [11], where electromagnetic loads were modeled in the first stage concept design of the DEMO divertor. Although material properties used in the study are missing from the report [11], thus preventing a direct (quantitative) comparison with the results given here, the main conclusion [11] suggested further enhancements of the first stage divertor concept such as introducing shunt paths for diverting the halo currents towards more massive structures. In this respect, the modeling inputs of the present analysis, accounting for a shunt element between the target plate and the cassette body, comply with the outcome of Ref. [11].

\subsection{Assumptions}

Analysis approximations:

- The effect of the external magnetic field is neglected in the calculation of the current distribution in a steady-state thermo-current analysis. 
- The change of the model geometry due to induced Lorentz magnetic forces in a steady-state mechanical analysis is assumed small enough not to affect the electrical current distributions.

Geometry:

- The geometry of the isolated-target divertor design follows the geometry of the standard divertor design with water cooling option 2 taken from WPDIV [12].

Materials (see also Tab. 1):

- The cassette body material is assumed to be Eurofer97 with isotropic electrical resistivity of $0.70 \mu \Omega \mathrm{m}$ (at $200^{\circ} \mathrm{C}$ ), isotropic elastic modulus of $200 \mathrm{GPa}$ and Poisson's ratio of 0.3 (all assumed temperature independent).

- The water-cooling pipes are assumed to be composed of two different materials [8]: $\mathrm{CuCrZr}$ in the PFC region and stainless steel AISI-316 in the non-PFC region (see Fig. 3), with isotropic electrical resistivity of $0.04 \mu \Omega \mathrm{m}\left(\right.$ at $200^{\circ} \mathrm{C}$ ) and $0.90 \mu \Omega \mathrm{m}\left(\right.$ at $\left.200^{\circ} \mathrm{C}\right)$, respectively. As the mono-block target is not explicitly modeled, both piping materials are assigned the same (generic) isotropic elastic modulus of $200 \mathrm{GPa}$ and Poisson's ratio of 0.3. Properties are assumed temperature independent.

- The water inside the pipes is assumed to be a perfect electrical insulator. This is a conservative assumption to estimate maximum current and stress amplitudes within the pipes (pure water resistivity ranges from 0.1 to $10 \mathrm{k} \Omega \mathrm{m}$ which is at least $10^{8}$ times higher than pipe resistivity).

Boundary conditions:

- In the thermo-current analysis zero electric potential is assigned to a node lying on a symmetry plane of the cassette model (or a node located furthest away from the target plates in the cooling pipes model). The divertor cassette and cooling pipes are assumed electrically isolated from the vacuum vessel (the effect of non-zero electrical connection between the cassette and vacuum vessel is discussed briefly in Sec. 3.3.3). 


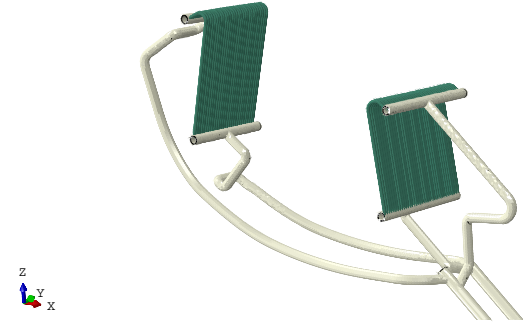

(a)

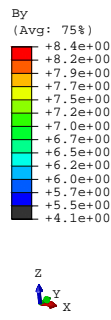

$\operatorname{Ls}_{x}^{x}$

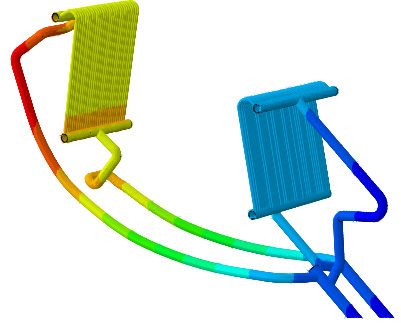

(b)

Figure 3: (a) Water-cooling pipes model used in the analysis with two assumed piping materials: $\mathrm{CuCrZr}$ in the PFC region (green) and stainless steel AISI-316 in the non-PFC region (grey). (b) Toroidal magnetic field distribution (By in $\mathrm{T}$ ) in the piping model.

- In the mechanical analysis the divertor cassette is fully clamped at the two ends to mimic the fixation of the supports. To model the fixation of the cooling pipes several regions along the pipes are assumed fully clamped (see orange arrows in Fig. 6(c)).

Loading conditions:

- Electrical current of $140 \mathrm{~A}$ (and $410 \mathrm{kA}$ - to model the most critical VDE current) is applied over the whole target plate area of the divertor cassette or cooling pipes.

- In the mechanical analysis the static body force $\mathbf{f}(\mathbf{r})=\mathbf{j}(\mathbf{r}) \times \mathbf{B}(\mathbf{r})$ is applied for radially decaying toroidal magnetic field $B(r)=B_{0} R_{0} / r$ acting on each element of the model with nominal field $B_{0}=5.667 \mathrm{~T}$ and major radius $R_{0}=9.072 \mathrm{~m}[16]$.

Finite element mesh:

- In both analyses the same FE mesh is used (with $42 \mathrm{k}$ elements for the cassette body model and 385k elements for the cooling pipes model), however, with different element types. Linear coupled thermal-electrical tetrahedrons (DC3D4E, with 4 Gauss points) and linear tetrahedrons (C3D4, with 1 Gauss point) are used in thermo-current and mechanical analyses, respectively. Both FE meshes have been tested to provide reasonably accurate results. 


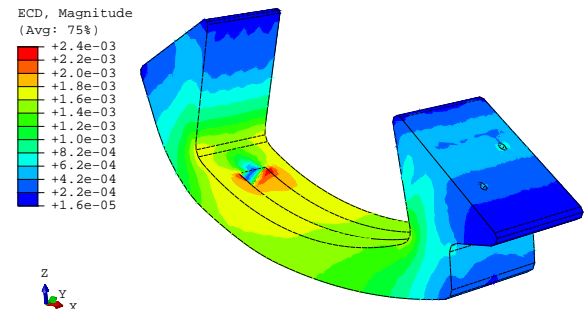

(a)

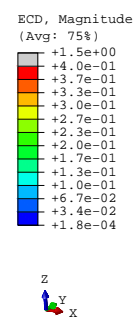

$L_{x}^{x}$

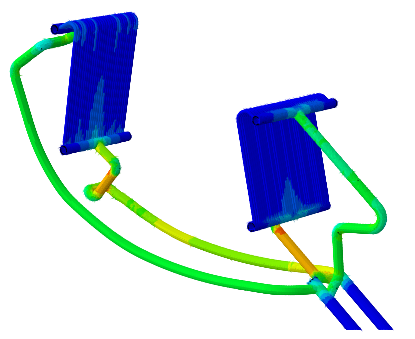

(c)

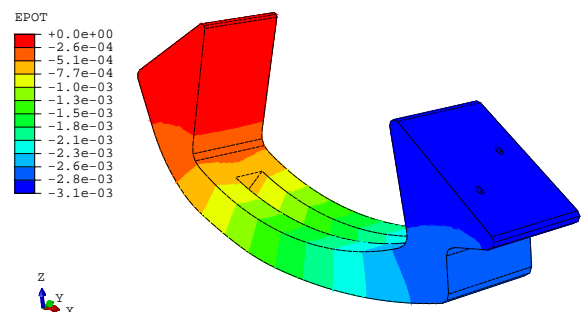

(b)

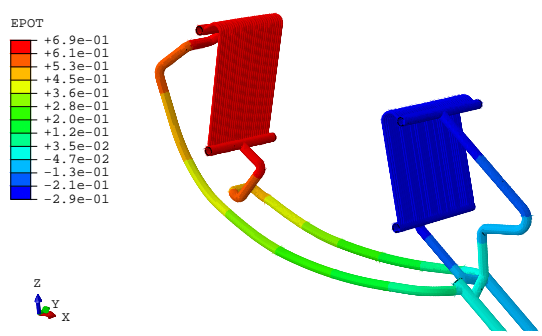

(d)

Figure 4: Results for the electrical current surface density (ECD in $\mathrm{A} / \mathrm{mm}^{2}$ ) and electrical potential (EPOT in V) for isolated divertor cassette ((a) and (b)) and isolated watercooling pipes ((c) and (d)) when $140 \mathrm{~A}$ is applied on the target region.

\section{RESULTS AND DISCUSSION}

\subsection{Current and potential distribution}

Figure 4 shows the calculated current densities (ECD in units of $\mathrm{A} / \mathrm{mm}^{2}$ ) and potential differences (EPOT in units of $\mathrm{V}$ ) along the divertor cassette and water-cooling pipes when a current of $140 \mathrm{~A}$ is applied separately on each of the component (assuming zero magnetic field). Both the cassette and cooling pipes are assumed isolated from the vacuum vessel. The maximum current density reaches $2.4 \mathrm{kA} / \mathrm{m}^{2}$ in the cassette body (located at the corners of the middle part) and approximately $0.40 \mathrm{MA} / \mathrm{m}^{2}$ in the cooling pipes (located in pipe sections below the target plates). The effective resistance of the cassette body is estimated to be $R_{c}=22 \mu \Omega$, with estimated maximum potential drop of $3.1 \mathrm{mV}$, and of the corresponding cooling piping $R_{p}=7.0 \mathrm{~m} \Omega$, with estimated maximum potential drop of $0.98 \mathrm{~V}$. The estimated power dissipated on the cassette body $(0.43 \mathrm{~W})$ and cooling pipes $(140 \mathrm{~W})$ is small enough to justify the neglected thermal effects. 


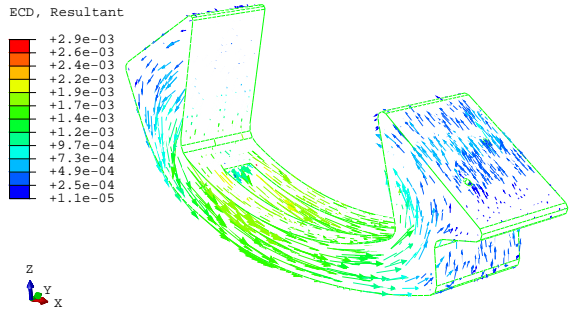

(a)

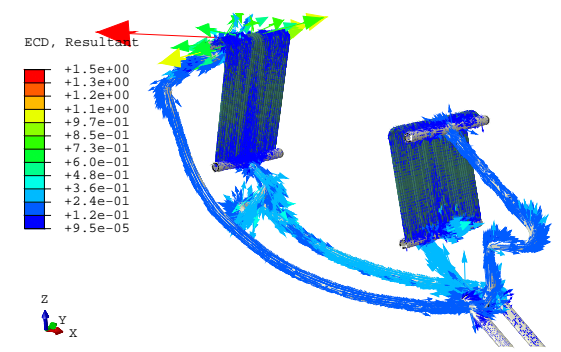

(c)

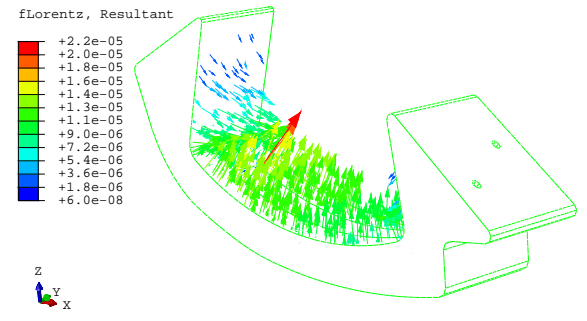

(b)

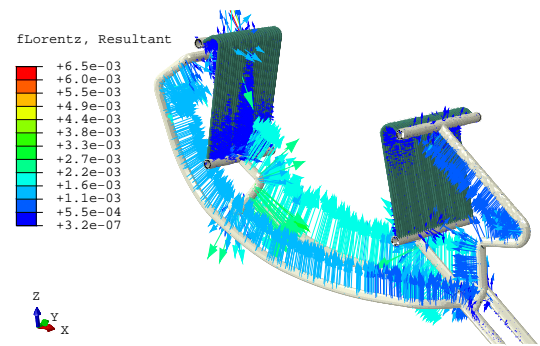

(d)

Figure 5: Vector fields of current surface density (ECD in $\mathrm{A} / \mathrm{mm}^{2}$ ) and Lorentz magnetic force density (fLorentz in $\mathrm{N} / \mathrm{mm}^{3}$ ) for isolated divertor cassette ((a) and (b)) and isolated water-cooling pipes ((c) and (d)) when $140 \mathrm{~A}$ is applied on the target region and $\mathrm{B}(\mathrm{r})$ is assumed in a toroidal direction (along y axis).

\subsection{Effect of external magnetic field}

The resulting distributions of electrical currents $\mathbf{j}(\mathbf{r})$, obtained for zero external magnetic field $(\mathrm{B}=0)$, are used next to calculate Lorentz magnetic forces using $\mathbf{f}(\mathbf{r})=\mathbf{j}(\mathbf{r}) \times \mathbf{B}(\mathbf{r})$ and radially decaying toroidal (along y axis) magnetic field $B(r)=B_{0} R_{0} / r$. Both vector fields, $\mathbf{j}(\mathbf{r})$ (ECD in units of $\mathrm{A} / \mathrm{mm}^{2}$ ) and $\mathbf{f}(\mathbf{r})$ (fLorentz in units of $\mathrm{N} / \mathrm{mm}^{3}$ ), are shown in Fig. 5 for better visualization. Lorentz force $\mathbf{f}(\mathbf{r})$ is applied as a body force on all the elements of the model to be used later in a mechanical analysis. It is important to note that results of Fig. 5 are approximate due to neglected coupling between the magnetic field and electrical currents (Hall effect). However, negligible error is expected from such an approximation.

\subsection{Mechanical stresses and displacements}

\subsubsection{Thermo current of $140 \mathrm{~A}$}

The results of the mechanical analyses are shown in Fig. 6. In overall, negligible von Mises stresses ( $\mathrm{S}$ in units of $\mathrm{MPa}$ ) and small displacements (U 


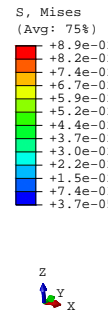

$L^{x}$

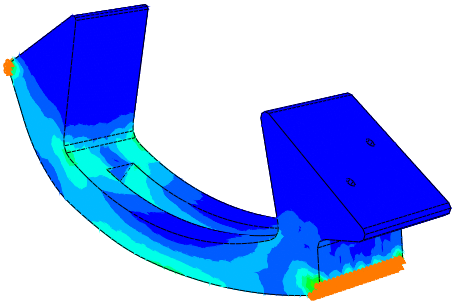

(a)

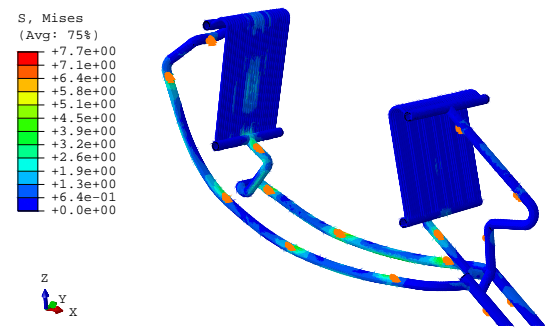

(c)

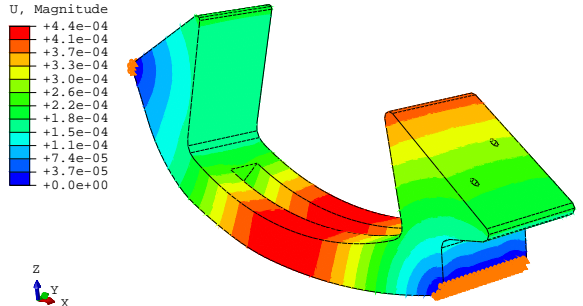

(b)
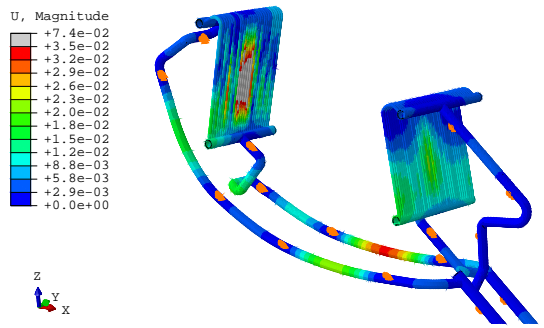

(d)

Figure 6: von Mises stress field ( $\mathrm{S}$ in $\mathrm{MPa}$ ) and displacement amplitude ( $\mathrm{U}$ in $\mathrm{mm}$ ) for isolated divertor cassette ((a) and (b)) and isolated water-cooling pipes ((c) and (d)) when $140 \mathrm{~A}$ is applied on the target plates and $\mathrm{B}(\mathrm{r})$ is assumed in a toroidal direction (along y axis). Orange arrows denote clamped boundary conditions modelling the supports and pipe fixation.

in units of $\mathrm{mm}$ ) are observed in both models. Typically, largest von Mises stresses appear at the locations of applied boundary conditions: approximately $90 \mathrm{kPa}$ in the cassette model and $8 \mathrm{MPa}$ in the cooling pipes model (to be compared with typical yield stress of AISI-316 stainless steel of minimum $200 \mathrm{MPa}$ at $200^{\circ} \mathrm{C}[17]$ ). For the latter case, it should be noted that even smaller stresses can be achieved when using better spatial arrangement (and/or larger number) of the fixations in the cooling pipe model. It is also assumed in the analysis that each such fixation is ideally isolating the pipe from the cassette body.

Small stresses also imply small displacements. The largest deformation of the cassette is observed in the middle section with $0.44 \mu \mathrm{m}$ upward deflection. However, considerably larger (but still small) displacements with maximum $35 \mu \mathrm{m}$ are identified in the middle section of the cooling pipes model. It should be noted that the largest pipe displacements (up to $74 \mu \mathrm{m}$ ) observed in the in-board PFC region in Fig. 6 (d) are not realistic and should be reduced 


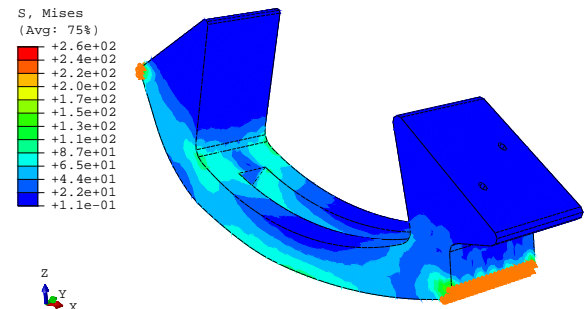

(a)

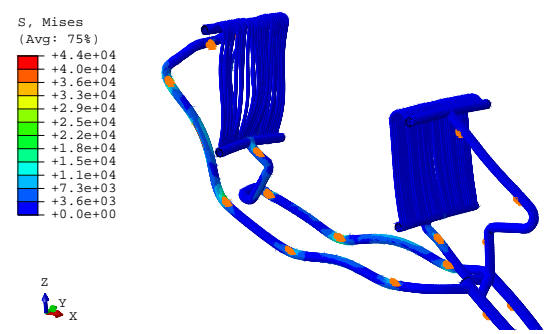

(c)

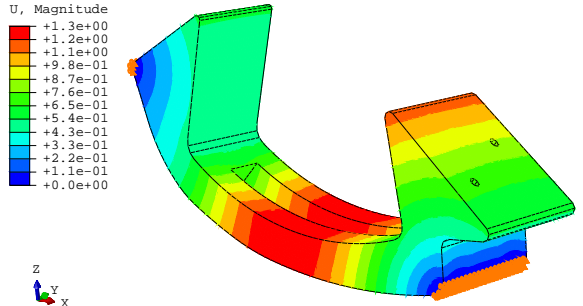

(b)

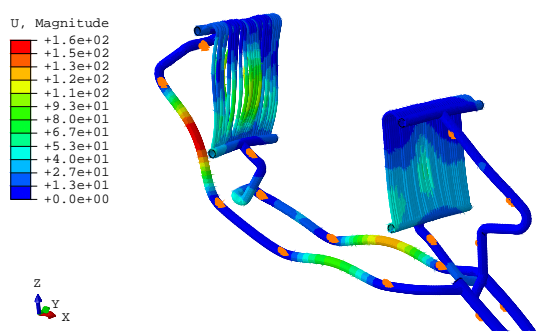

(d)

Figure 7: Same as Fig. 6 but using instead $410 \mathrm{kA}$ as the maximum VDE loading current.

considerably when accounting for the mono-block targets surrounding the pipes.

\subsubsection{VDE current of $410 \mathrm{kA}$}

Maximum current loading per divertor, generated during VDEs, can be conservatively estimated from the nominal plasma current of 19.6 MA redistributed evenly over 48 divertor modulus. In this way, a critical VDE current of $I_{V D E}=410 \mathrm{kA}$ per divertor is obtained. Note that a slightly lower value of $360 \mathrm{kA}$ was used in a similar electromagnetic force analysis for the divertor cassette [10] (but not for the water-cooling pipes) when using a 54 divertor design option.

Following the same procedure as before but using instead $410 \mathrm{kA}$ applied on the target plates, the stresses and displacements of the cassette are demonstrated in Figs. 7 (a) and (b). Due to linearity of the involved Ohm's and Hooke's laws, the obtained responses are, to a good approximation, magnified by factor $3000 \sim 410000 / 140$. The maximum von Mises stress of $260 \mathrm{MPa}$ (located in the clamped regions of the supports) is very close to the one presented in Ref. [10] (250 MPa). Also, the maximum vertical deflection 
of the cassette of $1.3 \mathrm{~mm}$ is close to the one reported in Ref. [10] (1.4 mm). The observed differences may be attributed to different current loadings but also to different material properties used in the analyses (not reported in Ref. [10]).

While maximum stresses in the divertor cassette are slightly above the allowed limits (inducing material yielding), the situation is much more serious in the cooling pipes model, see Figs. 7 (c) and (d). Namely, the observed maximum von Mises stress of approximately 35-45 GPa (located in the inboard vertical pipe region) is roughly 200 times higher than typical yield stress for AISI-316 steel and the corresponding displacements reach up to several tens of cm. Obviously, the applied $I_{V D E}=410 \mathrm{kA}$ current is devastating for the water-cooling pipes [11]. Going beyond the elastic material assumption, the realistic deflections of the cooling pipes would become even larger when accounting for elasto-plastic material behavior. Moreover, as the estimated electrical power dissipated on the cooling pipes is enormous (1.2 GW with the deposited energy of at least $80 \mathrm{MJ}$ over the assumed minimum current quench time of $70 \mathrm{~ms}$ [10]), the thermal effects should have been accounted for in the analysis.

\subsubsection{Admissible current through the water-cooling pipes}

The admissible upper current value for the water-cooling pipes $I_{p, \max }$ can be estimated from the results of Fig. 6 and using linear load-response assumption to obtain the maximum pipe stress equal to yield stress (minimum of $200 \mathrm{MPa}$ for AISI-316 steel at $200^{\circ} \mathrm{C}$ [17] and $\sim 200 \mathrm{MPa}$ for $\mathrm{CuCrZr}$ at $\left.200^{\circ} \mathrm{C}[15]\right)$. As already mentioned in Sec. 1, yield stress can be used as the simplest threshold criterion for damage initiation. In this way, following $140 \mathrm{~A} \cdot 200 \mathrm{MPa} / 8 \mathrm{MPa}$, the maximum allowed current through the water-cooling pipes is estimated to be $I_{p, \max }=3.5 \mathrm{kA}$. As maximum stresses usually emerge in the vicinity of pipe fixations in the non-PFC region, the obtained $I_{p, \max }$ is very strongly dependent on the number, shape and locations of these fixations. In this respect, the conservative value of $I_{p, \max }$ may be relatively easily increased by optimizing the pipe fixation system.

It is important to note that employing more realistic modelling of the PFC region, by accounting also mono-block targets surrounding the pipes, should further reduce the stresses in the PFC region in Fig. 6(c). In this regard, the onset of material yielding at $I_{p, \max }$ is expected to occur in the non-PFC piping region composed of AISI-316 steel.

In the isolated-target divertor design only a fraction of the current applied 
on the target plates actually flows through the water-cooling pipes (Fig. 1). This fraction is regulated by shunt resistance $R_{s}$ (Fig. 2). Assuming the extreme VDE scenario with $I_{V D E}=410 \mathrm{kA}$ applied on the target plates and $I_{p, \max }=3.5 \mathrm{kA}$, a conservative upper value for shunt resistance is readily obtained following Eq. (4), $R_{s, \max }=38 \mu \Omega$.

In the more realistic case of finite electrical connection between the divertor cassette and the vacuum vessel (e.g., via electrically conductive fixation supports), part of the cassette current would subdivide and flow also through the vacuum vessel. In this respect, the effective resistance of the cassette $R_{c}$ would reduce to $\alpha R_{c}$ with $\alpha\left(\alpha_{\min }<\alpha<1\right)$ accounting for the resistance of the fixation supports and vacuum vessel (for a very good electrical connection $\alpha_{\text {min }} \sim 0.5$ can be estimated). According to the electrical circuit diagram shown in Fig. 2, the reduction of $R_{c}$ also implies the reduction of the pipe current $I_{p}$ (assuming constant $I, R_{s}$ and $R_{p}$ ), which finally results also in smaller pipe stresses. For $R_{p} \gg R_{c}+R_{s}$, the $I_{p}$ would reduce to approximately $\beta I_{p}$ where $\beta=\left(\alpha R_{c}+R_{s}\right) /\left(R_{c}+R_{s}\right)$. Assuming also $R_{s} \sim 2 R_{c}$ and good electrical connection between the cassette and the vessel $\left(\alpha_{\min } \sim 0.5\right)$, $\beta_{\min } \sim\left(2+\alpha_{\text {min }}\right) / 3 \sim 0.83$, which results in up to $17 \%$ reduction of the pipe stresses.

In a similar way, assuming effective cassette resistance $\alpha R_{c}$ in the case of finite electrical connection between the divertor cassette and the vacuum vessel, the conservative upper value for shunt resistance, $R_{s, \max }=38 \mu \Omega$, can be increased (see Eq. (4)) to $R_{s, \max }+(1-\alpha) R_{c}$, which is bounded by $R_{s, \text { max }} \sim 49 \mu \Omega$ for $\alpha_{\text {min }} \sim 0.5$.

\subsection{Thermo-current measurement resolution}

For ASDEX Upgrade a total thermo-current at the target of $1870 \mathrm{~A}$ is predicted for $3 \mathrm{MW}$ flowing over the separatrix assuming $\lambda_{q}=2 \mathrm{~mm}$, where $\lambda_{q}$ is the power fall-off length at the divertor plate (Ref. [4]). This will result in a current of $15 \mathrm{~A}$ for each of the 128 tiles in the circumference. The shunt resistances are $2 \mathrm{~m} \Omega$, so that the signal of $30 \mathrm{mV}$ is expected. This corresponds to 200 bits of a $+/-5 \mathrm{~V} 16$ bit ADC. Shown in Fig. 8 is the example of a plasma discharge with shunt resistance measurements in ASDEX Upgrade. The bandwidth of the isolation amplifier (AD 215) is $100 \mathrm{kHz}$ so that the time resolution is sufficient to resolve ELM's. The control parameter, Tdiv, used for impurity feedback experiments to limit the power flux to the divertor plate is produced by using a median value of the shunt 


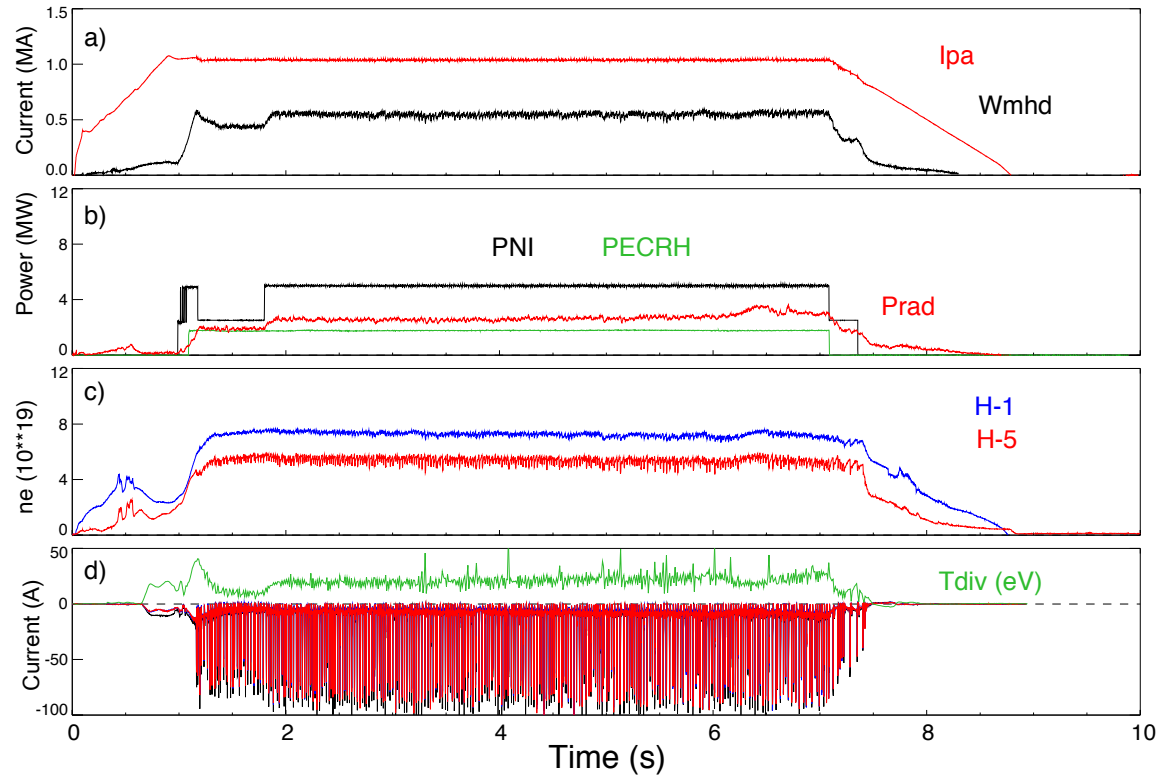

Figure 8: Overview of a discharge showing the time evolution of a) plasma current (Ipa) and plasma energy (Wmhd), b) the neutral beam injection heating power (PNI), electron cyclotron heating power (PECRH) and radiated power (Prad), c) the electron density line integral in the core (H-1) and edge (H-5) of the plasma and d) the currents to the outer divertor with negative polarity from the shunt measurements at 3 toroidal positions. A scaled median is taken at $1 \mathrm{~ms}$ intervals to produce the signal, Tdiv, without ELM spikes for power flux control to the divertor.

resistance measurement, so that excursions due to ELM's can be removed [18].

In the case of plasma detachment measurement in DEMO, the applied thermo-current of $I=140$ A would induce shunt voltage of $U_{s}=5.3 \mathrm{mV}$ assuming $R_{s}=38 \mu \Omega$, see Eq. (3). In a rather more realistic case, with assumed good electrical connection between the divertor cassette and the vacuum vessel, the applied thermo-current of $I=140$ A would induce shunt voltage up to $U_{s} \sim 6.8 \mathrm{mV}$ for the assumed $R_{s} \sim 49 \mu \Omega$. In either case, this is approximately an order of magnitude smaller than the signals available for power flux control to protect the divertor plates in ASDEX Upgrade. An 
increase in the measured shunt voltage in DEMO by increasing the shunt resistance can only be realized if the water cooling pipe resistance could be increased at the same time to ensure that the limit of $\sim 3.5 \mathrm{kA}$ current flowing through the water cooling pipe in a disruption could be maintained. Limiting the bandwidth of the Tdiv signal could be another means of obtaining sufficient resolution for divertor power flux control by thermo-current shunt measurements. Alternatively, increasing the cassette resistance to $60 \mu \Omega$ $\left(R_{c}+R_{s}\right.$ in Fig. 2) and measuring the potential difference of the inner and outer divertor target plates is suggested as a possible means of maximizing the thermo-current shunt signal. However, the quality of the measured potential difference could be deteriorated by the long single cables to each plasma facing component which then form a pick up loop with the cassette.

\section{CONCLUSIONS}

Finite-element analysis has been performed to calculate a conservative upper value for shunt resistance in the isolated-target design solution of the DEMO divertor. This value (estimated to $\sim 40 \mu \Omega$ ) has been derived from the requirement that, in the case of plasma disruption event (VDE), a fraction of the generated halo current flowing through the water-cooling pipes reaches the critical value (estimated to $\sim 3.5 \mathrm{kA}$ ) at which local pipe yielding develops due to induced electromagnetic forces. The calculated shunt resistance

provides a thermo-current shunt voltage in the range of few millivolts which seems to be on the lower limit for reliable plasma detachment detection.

\section{ACKNOWLEDGMENTS}

This work has been carried out within the framework of the EURO fusion Consortium and has received funding from the Euratom Research and Training Programme 2014-2018 under grant agreement No. 633053. The views and opinions expressed herein do not necessarily reflect those of the European Commission. S.E.S. also gratefully acknowledges the financial support from the Slovenian Research Agency through research program P2-0026.

\section{References}

[1] A. Kallenbach, et al., "Optimized tokamak power exhaust with double radiative feedback in ASDEX Upgrade", Nucl. Fusion 52, 122003, 2012. 
[2] G. Pautasso, et al., "The halo current in ASDEX Upgrade", Nucl. Fusion 51, 043010, 2011.

[3] P. J. Harbour et. al, "The X-point scrape-off plasma in JET with L- and H-modes", J. Nucl. Materials 236, 162, 1989.

[4] A. Kallenbach, et al., "Electric currents in the scrape-off layer in ASDEX Upgrade", J. Nucl. Materials 639, 290-293, 2001.

[5] G. M. Staebler and F.L. Hinton, "Currents in the scrape-off layer of diverted tokamaks", Nucl. Fusion 29, 1820, 1989.

[6] A. V. Chankin, et al., "Parallel currents in the scrape-off layer of JET diverted discharges", J. Nucl. Materials 196, 739, 1992.

[7] L. Giannone, S. El Shawish, "Divertor thermo-current measurements and initial CAD studies and engineering analysis, including diagnostic integration on DEMO", final report, 2016, EFDA_D_2N8KBA.

[8] L. Giannone, et al., "Conceptual study for detachment measurements in DEMO", final report, 2017, (EFDA number not available).

[9] Simulia, 2015, ABAQUS 6.14-2.

[10] J. H. You, et al., "Progress in the initial design activities for the European DEMO divertor: Subproject Cassette", Fus. Eng. Des. 124, 364, 2017.

[11] V. Cocilovo, G. Ramogida, "Modeling of Electromagnetic Loads on DEMO First Stage Divertor", Fus. Sci. Tech., 72, 478, 2017.

[12] J. H. You, et al., "Divertor cassette design development", final report, 2015, EFDA_D_2MGK4L.

[13] K. Mergia, N. Boukos, "Structural, thermal, electrical and magnetic properties of Eurofer 97 steel", J. Nuc. Mat., 373, 1, 2008.

[14] T. K. Chu, C. Y. Ho, "Thermal Conductivity and Electrical Resistivity of Eight Selected AISI Stainless Steels." In: Mirkovich V.V. (eds) Thermal Conductivity 15. Springer, Boston, MA, 1978. 
[15] I. S. Batra, et al. "Microstructure and properties of a $\mathrm{CuCrZr}$ alloy", J. Nuc. Mat., 299, 91, 2001.

[16] V. Cocilovo, G. Ramogida, "Simulation of electromagnetic VDE plasma effects on divertor structures of DEMO", Fus. Eng. Des., in press, 2018.

[17] http://www.atlassteels.com.au/

[18] A Kallenbach et al.,"Divertor power load feedback with nitrogen seeding in ASDEX Upgrade", Plasma Phys. Control. Fusion, 52, 055002, 2010. 